\title{
Supporting Information \\ Effects of 1-methylcyclopropene (1-MCP) and Ethylene on Postharvest Lignification of Common Beans (Phaseolus \\ vulgaris $\mathbf{L}$ )
}

Guofang Xie ${ }^{\mathrm{a}, \mathrm{b}}$, Yingchun Feng ${ }^{\mathrm{b}}$, Yao Chen ${ }^{\mathrm{b}}$, Mingsheng Zhang a,"

${ }^{a}$ Key laboratory of Plant Resource Conservation and Germplasm Innovation in Mountainous Region (Ministry of Education), Collaborative Innovation Center for Mountain Ecology \& Agro-Bioengineering (CICMEAB), College of Life Sciences/Institute of Agro-bioengineering, Guizhou University, Guiyang 550025, Guizhou Province, China

${ }^{b}$ Food and Pharmaceutical Engineering Institute/Guizhou Engineering Research Center for Fruit Processing, Guiyang University, Guiyang 550005, Guizhou, China

*Corresponding Author: Mingsheng Zhang |E-mail: mshzhang@163.com (Mingsheng. Zhang). 


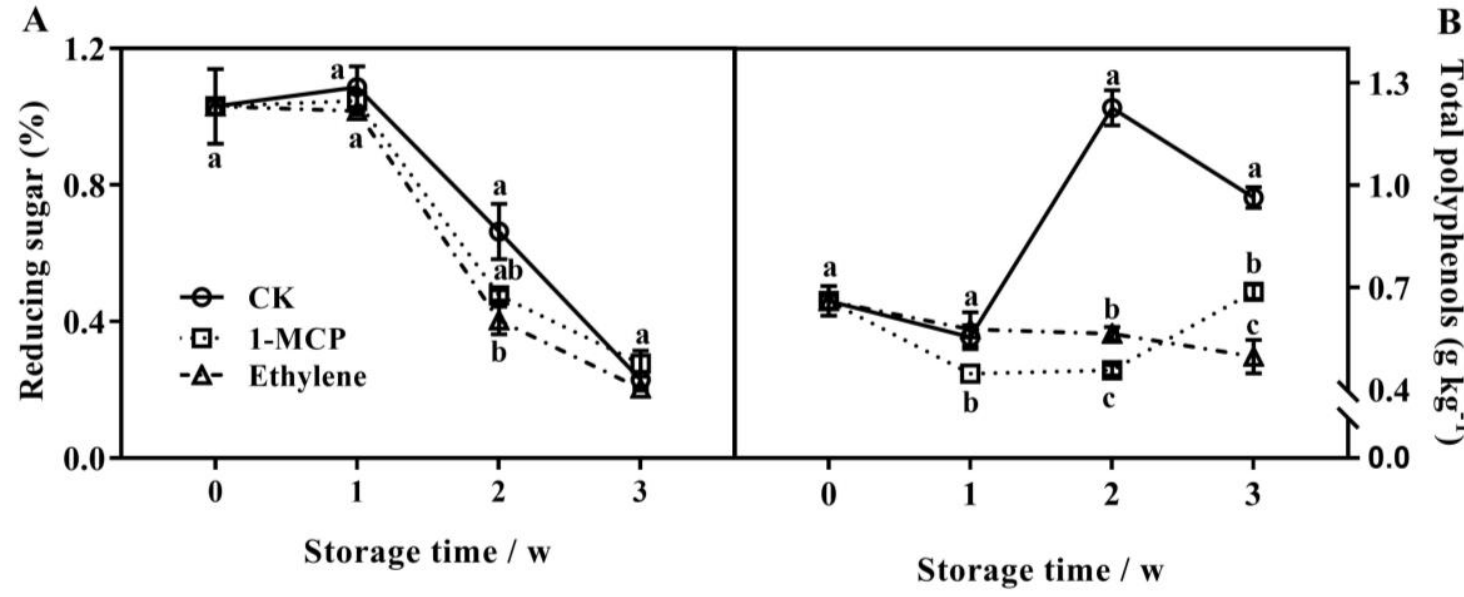

Figure S1: (A)Firmness and (B) reducing sugar of Control, Ethylene and 1-MCP treated beans during storage. Vertical bars represent standard error of three replicates $(n=10)$. Different letters within each parameter indicate statistically significant differences $(p<0.05)$

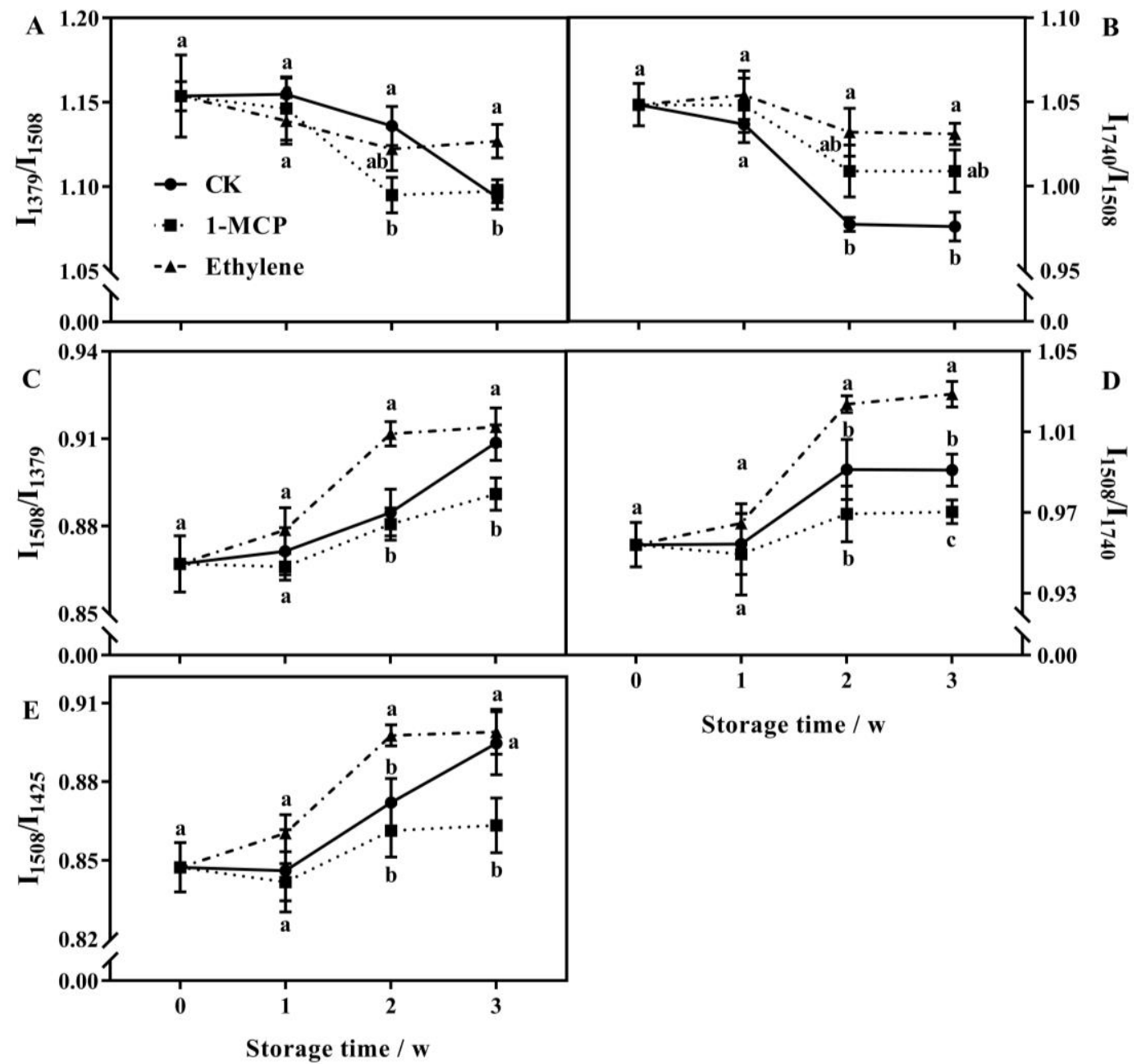

Figure S2: The ratio (A) $\mathrm{I}_{1379} / \mathrm{I}_{1508}$, (B) $\mathrm{I}_{1740} / \mathrm{I}_{1508}$, (C) $\mathrm{I}_{1508} / \mathrm{I}_{1379}$, (D) $\mathrm{I}_{1508} / \mathrm{I}_{1425}$, and (E) $\mathrm{I}_{1508} / \mathrm{I}_{1740}$, of FTIR characteristic peak height of Control, Ethylene and 1-MCP treated beans during storage. Vertical bars represent standard error of three replicates $(n=32)$. Different letters within each parameter indicate statistically significant differences $(p<0.05)$ 


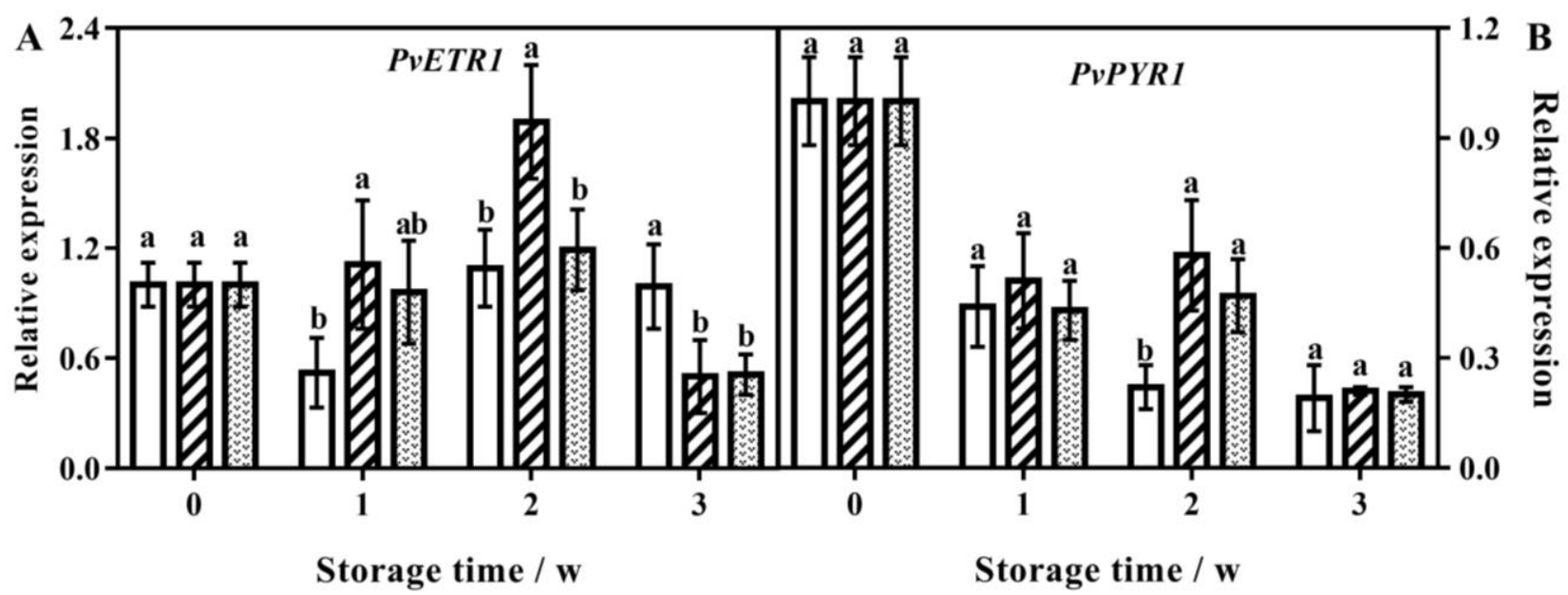

Figure S3: Expressions of $P v E T R 1$ (A) and $P \nu$ PYR1 (B) genes of Control, Ethylene and 1-MCP treated beans during storage. Samples from Day 0 (assigned an arbitrary quantity of " 1 ") were used as a calibrator to calculate the relative quantity of the results. Vertical bars represent standard error of three replicates (10 beans of each). Different letters within each parameter indicate statistically significant differences $(P<0.05)$

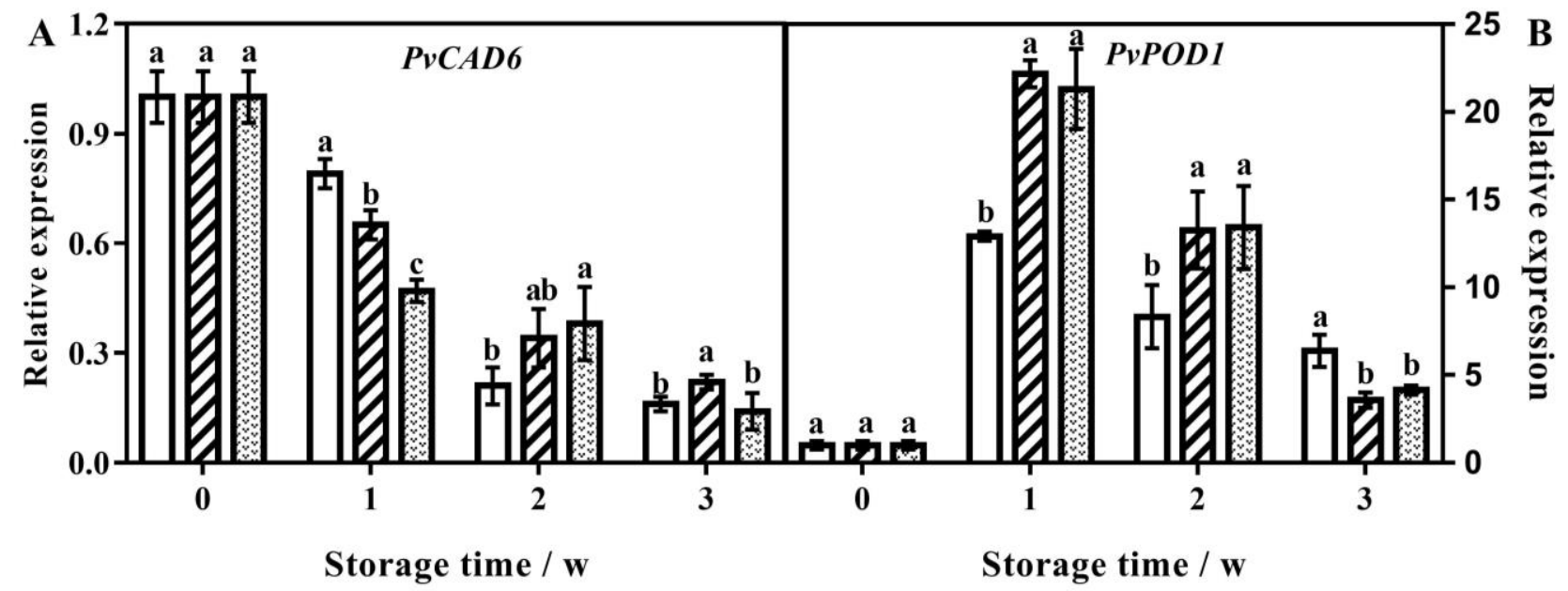

Figure S4: Expressions of $P v C A D 6$ (A) and $P v$ POD1 (B) genes of Control, Ethylene and 1-MCP treated beans during storage. Samples from Day 0 (assigned an arbitrary quantity of "1") were used as a calibrator to calculate the relative quantity of the results. Vertical bars represent standard error of three replicates (10 beans of each). Different letters within each parameter indicate statistically significant differences $(P<0.05)$ 


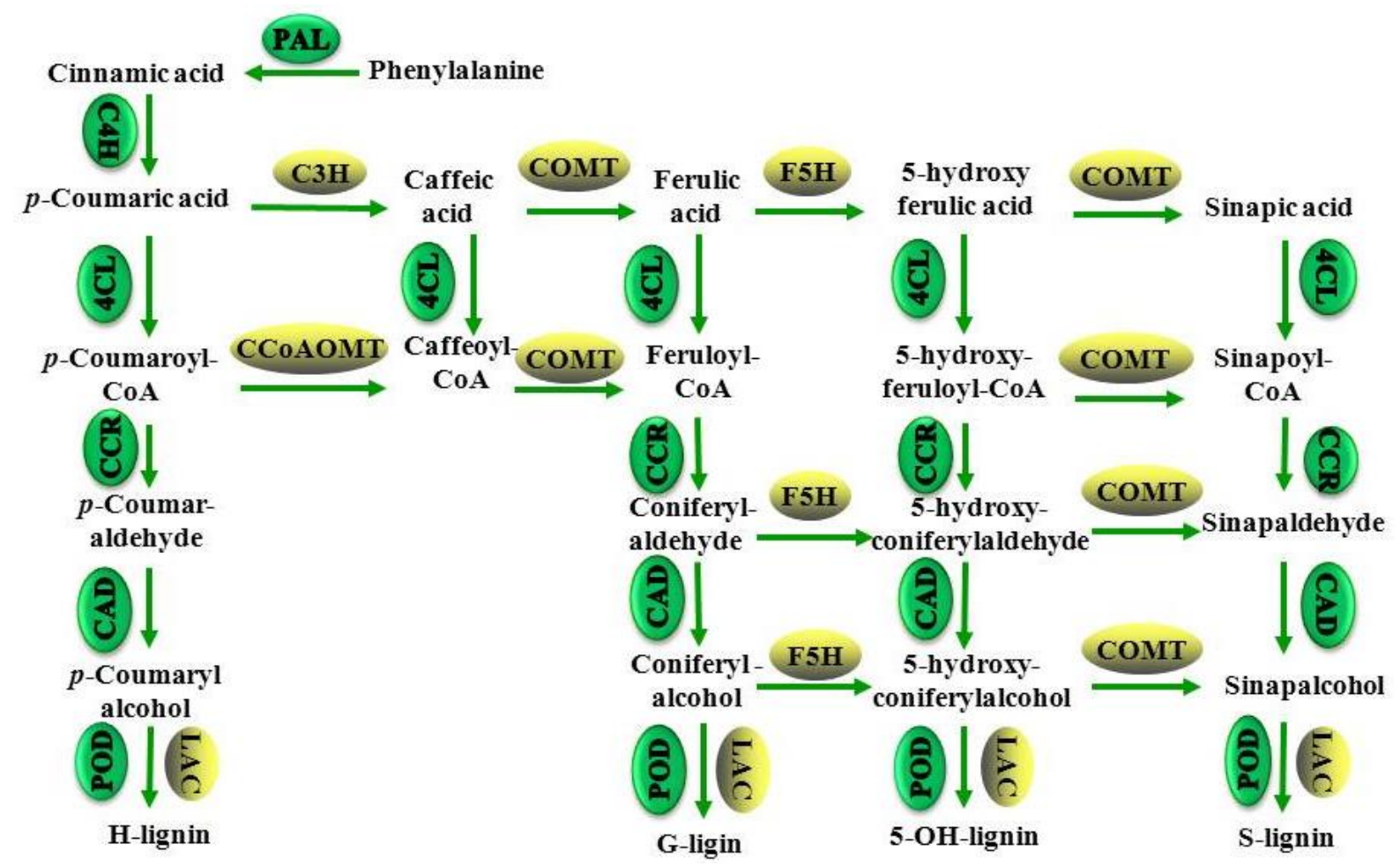

Figure S5: Simplified scheme of the lignin biosynthetic pathway in celery plant. Twelve enzymes are involved in this pathway including phenylalanine ammonialyase (PAL), cinnamate 4-hydroxylase $(\mathrm{C} 4 \mathrm{H})$, 4-coumarateCoA ligase $(4 \mathrm{CL})$, p-coumaroyl shikimate/quinate 3 '-hydroxylase $(\mathrm{C} 3 \mathrm{H})$, caffeoyl-CoA O-methyl-transferase (CCoAOMT), ferulate 5-hydroxylase (F5H), caffeic acid O-methyltransferase (COMT), cinnamoyl-CoA reductase (CCR), cinnamyl alcohol dehydrogenase $(\mathrm{CAD})$, peroxidase (POD), and laccase (LAC).

Table S1: Primers used in the qRT-PCR analysis

\begin{tabular}{|c|c|c|c|}
\hline Gene & Gene ID & Forward primer (5'-3') & Reverse primer (5'-3') \\
\hline$P v \mathrm{ACO} 1$ & 18639833 & ATGGAGCAAAGGTTCAAG & GATGTTGGAATCTGGAAGA \\
\hline$P v$ ETR1 & 18616939 & GCAAGAAGAGAAGCAGAA & TCCTGTAGTAACGAAGAGA \\
\hline$P \nu \mathrm{PYR} 1$ & 18621272 & ATGCCTGATGCCATTAAG & CAATTCTATGCTTCAAGTGTT \\
\hline$P v A O G 1$ & 18633901 & TTCGTGGTTCCTAATCTTC & TCTCCTCTAACTGCTTCA \\
\hline$P v \operatorname{SuSy} 2$ & 18621256 & GACAATATGAGAGCCACAGT & GCACCAGGAGACACAATA \\
\hline$P v \mathrm{PAL} 3$ & 18641535 & CTGGCACGACATCATAAGC & GGAGGTGGTGGTGTTGTA \\
\hline$P v 4 \mathrm{CL} 1$ & 18615556 & AATAGTGACTGTGGATAC & TGTGGATTGTTGTGATAA \\
\hline PvCOMT1 & 18621334 & GATGAACAGTGCGTGAAG & AGTCCACCACAATAACCTT \\
\hline$P \nu \mathrm{CAD} 6$ & 18639950 & AACATAACGAGTGACATTGAAT & AACGGTAGCGAACATCAT \\
\hline$P v \mathrm{POD} 1$ & 18622267 & AAATGGTTGCCCTCTCAG & ATCTATGTTGGTGTCGTTGTA \\
\hline
\end{tabular}

